\title{
Biomimetic Total Synthesis of Litseaverticillols B, E, I, and J, and Structural Reassignment of Litseaverticillol E.
}

Georgios Vassilikogiannakis*, loannis Margaros, Tamsyn Montagnon

Department of Chemistry, University of Crete, 71409 Iraklion, Crete, Greece

vasil@chemistry.uoc.gr 
Supporting Information Table of Contents:

\begin{tabular}{|c|c|}
\hline Spectrum title & $\begin{array}{c}\text { Page } \\
\text { number }\end{array}$ \\
\hline${ }^{1} \mathrm{H} \mathrm{NMR}\left(500 \mathrm{MHz}, \mathrm{CDCl}_{3}\right)$ spectrum of lactone 8 & 3 \\
\hline${ }^{13} \mathrm{C} \mathrm{NMR}\left(125 \mathrm{MHz}, \mathrm{CDCl}_{3}\right)$ spectrum of lactone 8 & 4 \\
\hline${ }^{1} \mathrm{H}$ NMR $\left(500 \mathrm{MHz}, \mathrm{CDCl}_{3}\right)$ spectrum of furan 9 & 5 \\
\hline${ }^{13} \mathrm{C} \mathrm{NMR}\left(125 \mathrm{MHz}, \mathrm{CDCl}_{3}\right)$ spectrum of furan 9 & 6 \\
\hline${ }^{1} \mathrm{H}$ NMR $\left(500 \mathrm{MHz}, \mathrm{CDCl}_{3}\right.$ ) spectrum of unpurified hydroperoxide $\mathbf{1 1}$ & 7 \\
\hline${ }^{13} \mathrm{C}$ NMR $\left(125 \mathrm{MHz}, \mathrm{CDCl}_{3}\right)$ spectrum of unpurified hydroperoxide $\mathbf{1 1}$ & 8 \\
\hline${ }^{1} \mathrm{H}$ NMR $\left(500 \mathrm{MHz}, \mathrm{CDCl}_{3}\right)$ spectrum of litseaverticillol B (1) & 9 \\
\hline${ }^{13} \mathrm{C} \mathrm{NMR}\left(125 \mathrm{MHz}, \mathrm{CDCl}_{3}\right)$ spectrum of litseaverticillol B (1) & 10 \\
\hline $\begin{array}{l}{ }^{1} \mathrm{H} \text { NMR }\left(500 \mathrm{MHz}, \mathrm{CDCl}_{3}\right) \text { spectrum of proposed structure } \\
\text { litseaverticillol E (2) }\end{array}$ & 11 \\
\hline $\begin{array}{l}{ }^{3} \mathrm{C} \text { NMR }\left(125 \mathrm{MHz}, \mathrm{CDCl}_{3}\right) \text { spectrum of proposed structure } \\
\text { litseaverticillol E (2) }\end{array}$ & 12 \\
\hline${ }^{1} \mathrm{H}$ NMR (500 MHz, $\mathrm{CDCl}_{3}$ ) spectrum of litseaverticillol I (4) & 13 \\
\hline${ }^{13} \mathrm{C} \mathrm{NMR}\left(125 \mathrm{MHz}, \mathrm{CDCl}_{3}\right)$ spectrum of litseaverticillol I (4) & 14 \\
\hline${ }^{1} \mathrm{H}$ NMR $\left(500 \mathrm{MHz}, \mathrm{CDCl}_{3}\right)$ spectrum of litseaverticillol J (5) & 15 \\
\hline${ }^{13} \mathrm{C} \mathrm{NMR}\left(125 \mathrm{MHz}, \mathrm{CDCl}_{3}\right)$ spectrum of litseaverticillol J (5) & 16 \\
\hline $\begin{array}{l}{ }^{\mathrm{l}} \mathrm{H} \mathrm{NMR}\left(500 \mathrm{MHz}, \mathrm{CDCl}_{3}\right) \text { spectrum of revised structure } \\
\text { litseaverticillol E (3) }\end{array}$ & 17 \\
\hline $\begin{array}{l}{ }^{13} \mathrm{C} \text { NMR }\left(125 \mathrm{MHz}, \mathrm{CDCl}_{3}\right) \text { spectrum of revised structure } \\
\text { litseaverticillol } \mathrm{E} \mathrm{(3)}\end{array}$ & 18 \\
\hline HRMS for litseaverticillol E (3, revised structure) & 19 \\
\hline
\end{tabular}




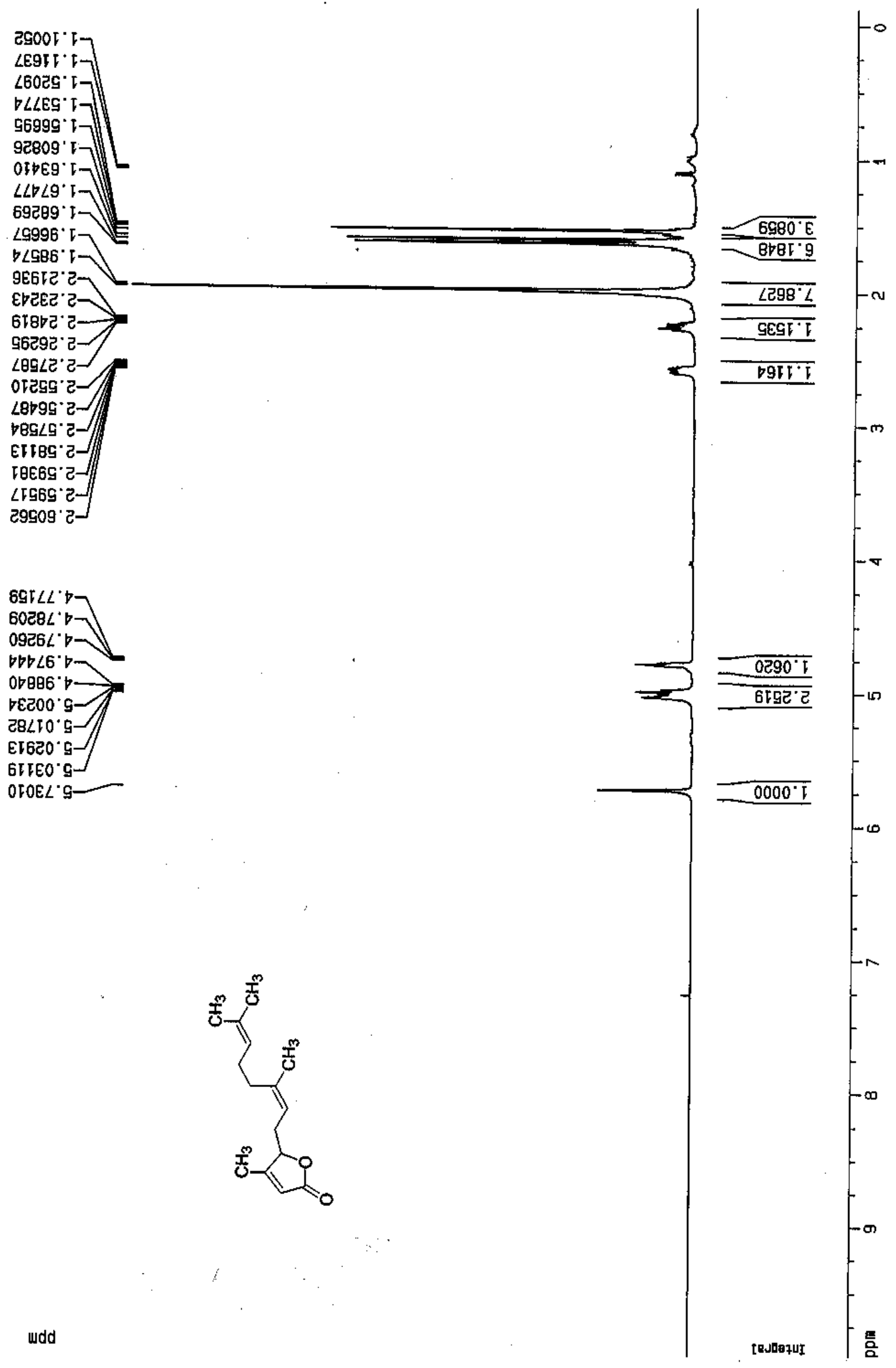


C $\angle 0^{\circ} \mathrm{EL}$

$299^{\circ} \angle \mathrm{V}$

EFE' $\varepsilon 2$

ट己g'g己 -

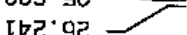

EBO $0 \mathrm{E}-$

$090^{\circ} \mathrm{CE}$

506. $9 L$

9gF $L \longrightarrow$

IIt $\angle L$

09ट" 60

098. 915

$\angle 9 V^{\circ} \angle F F>$

EPE"ECI-

E6LL IEI - -

E89.6ET-

$D \Rightarrow E^{\circ} \mathrm{BST}$

$\angle \triangle 0^{\circ} E \angle T \ldots$

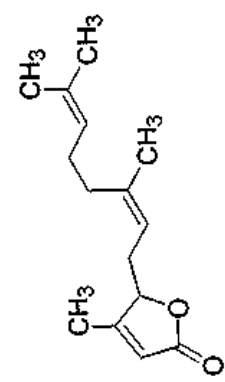

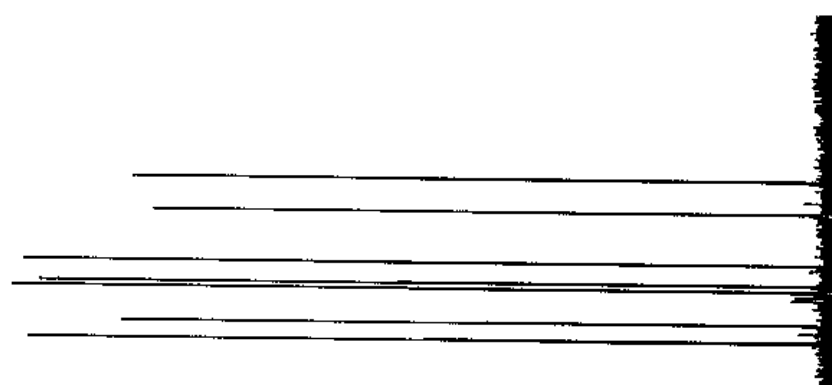

o
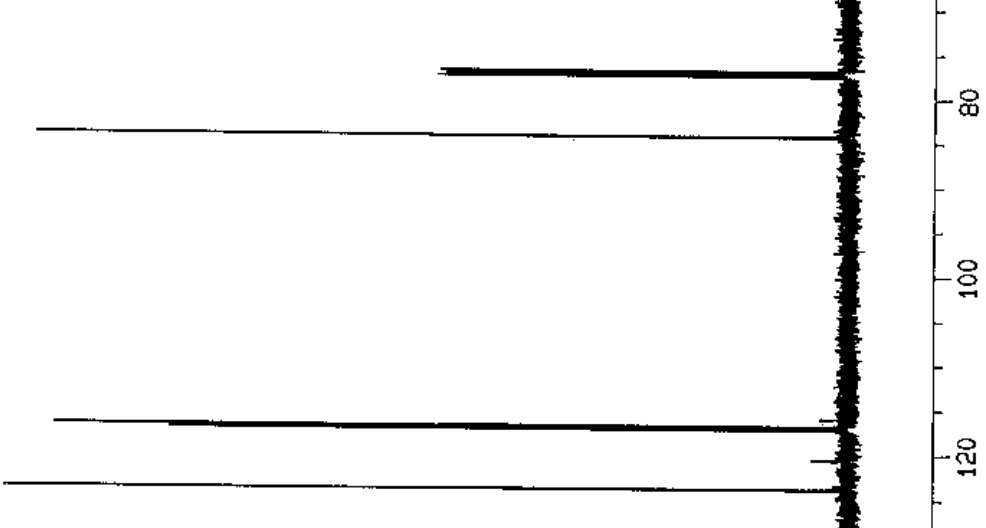


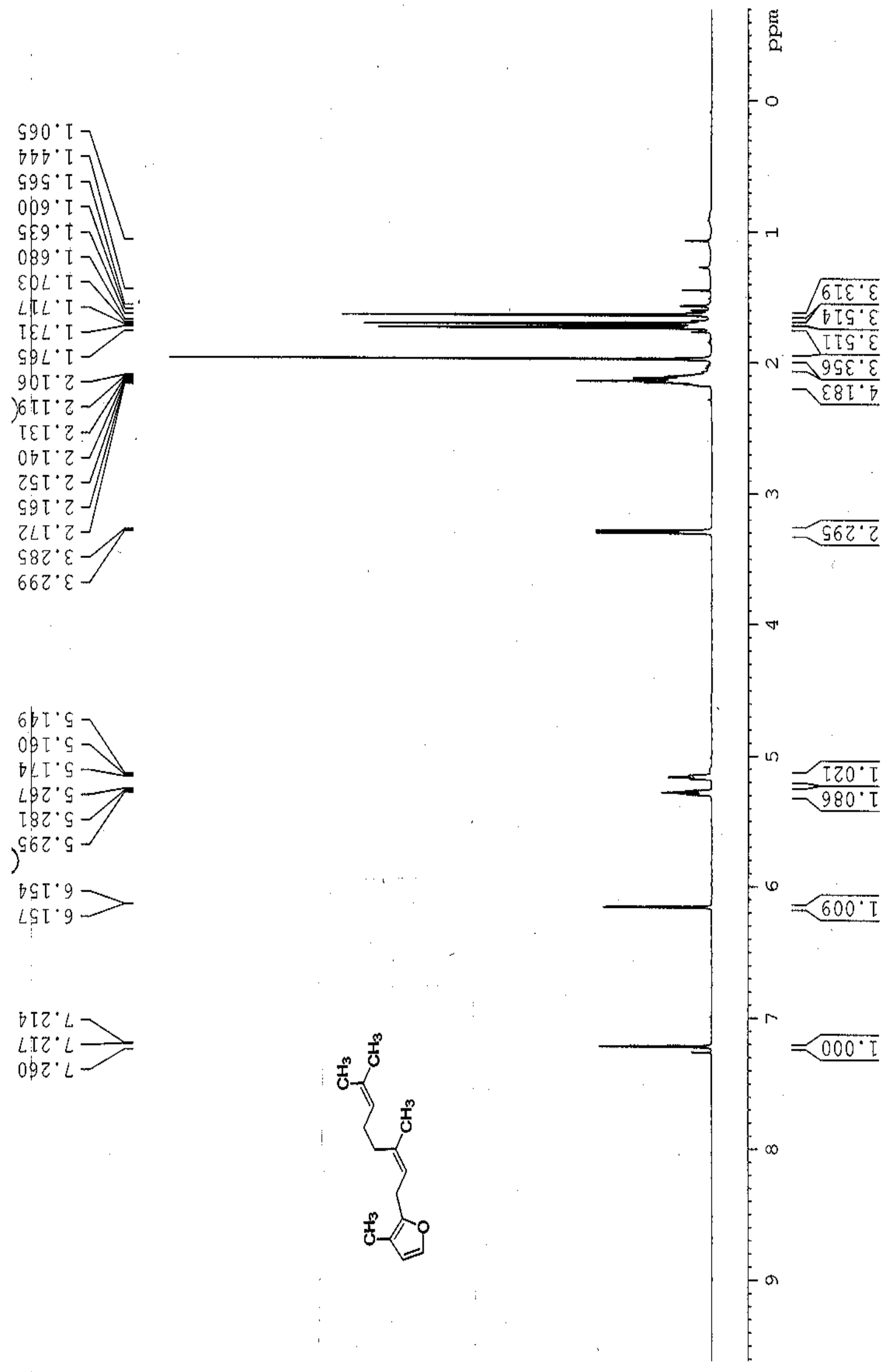




$$
1
$$




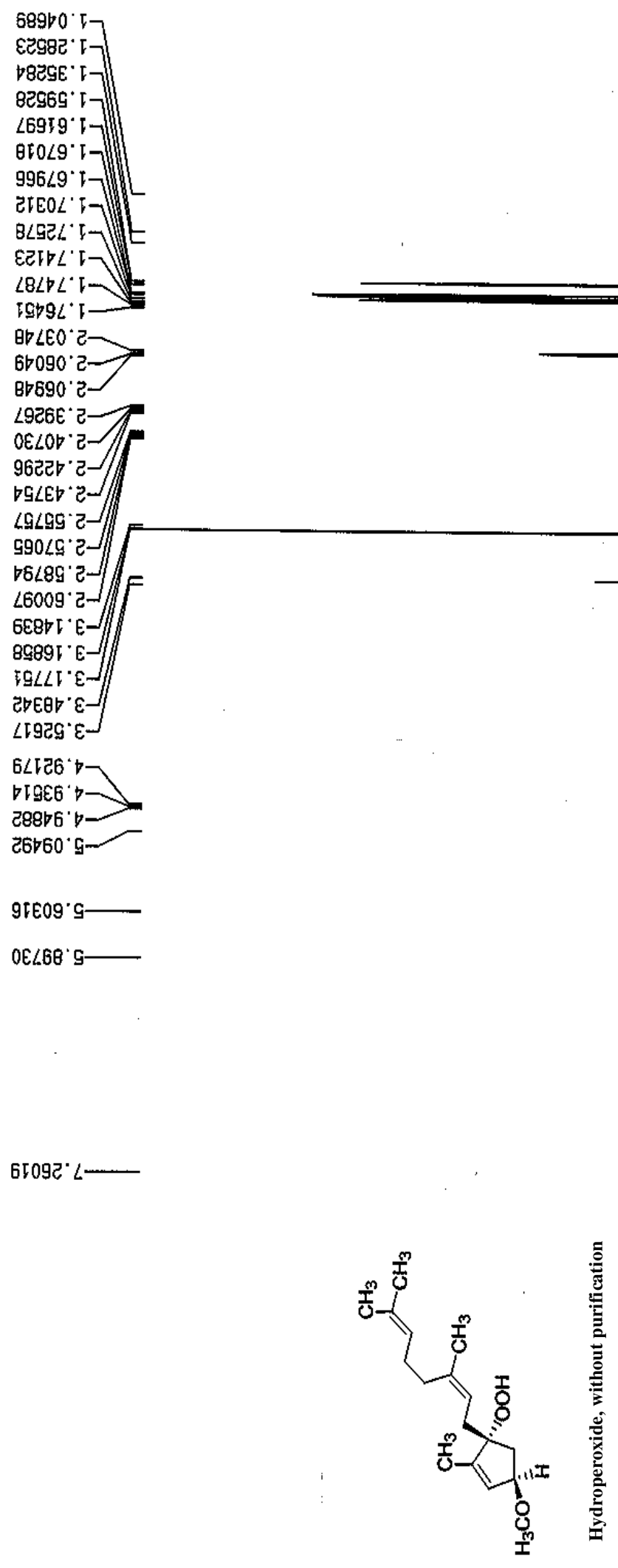

wdd

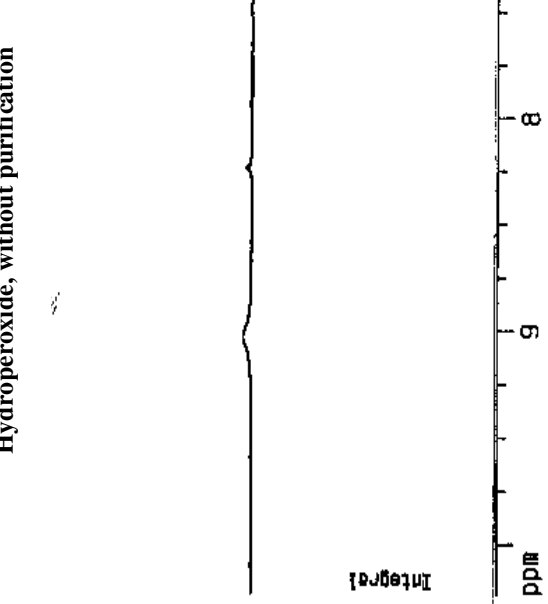


VDL'T

ट29. $\angle T=$

$999^{\circ} \varepsilon 2$

B $\angle 9 \cdot 92 \longrightarrow$

ㅂㄹㄱ' 92

gbe' $2 \varepsilon-$

ट己ᄅ' $\mathrm{ge} \longrightarrow$

I2r.09

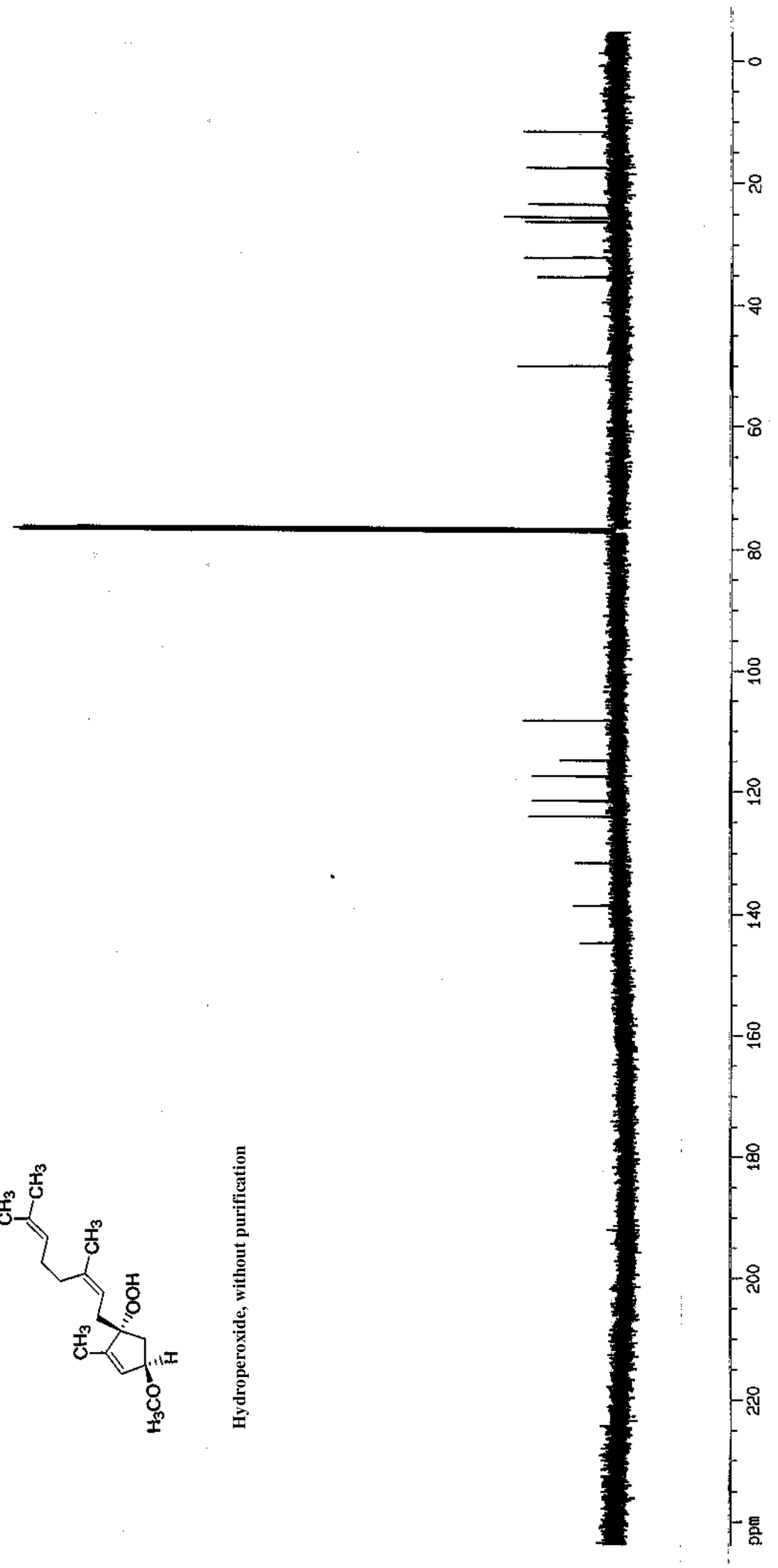

$9 \forall \angle ' 9 L$

$000 \angle \longrightarrow$
$\operatorname{tgc} L$

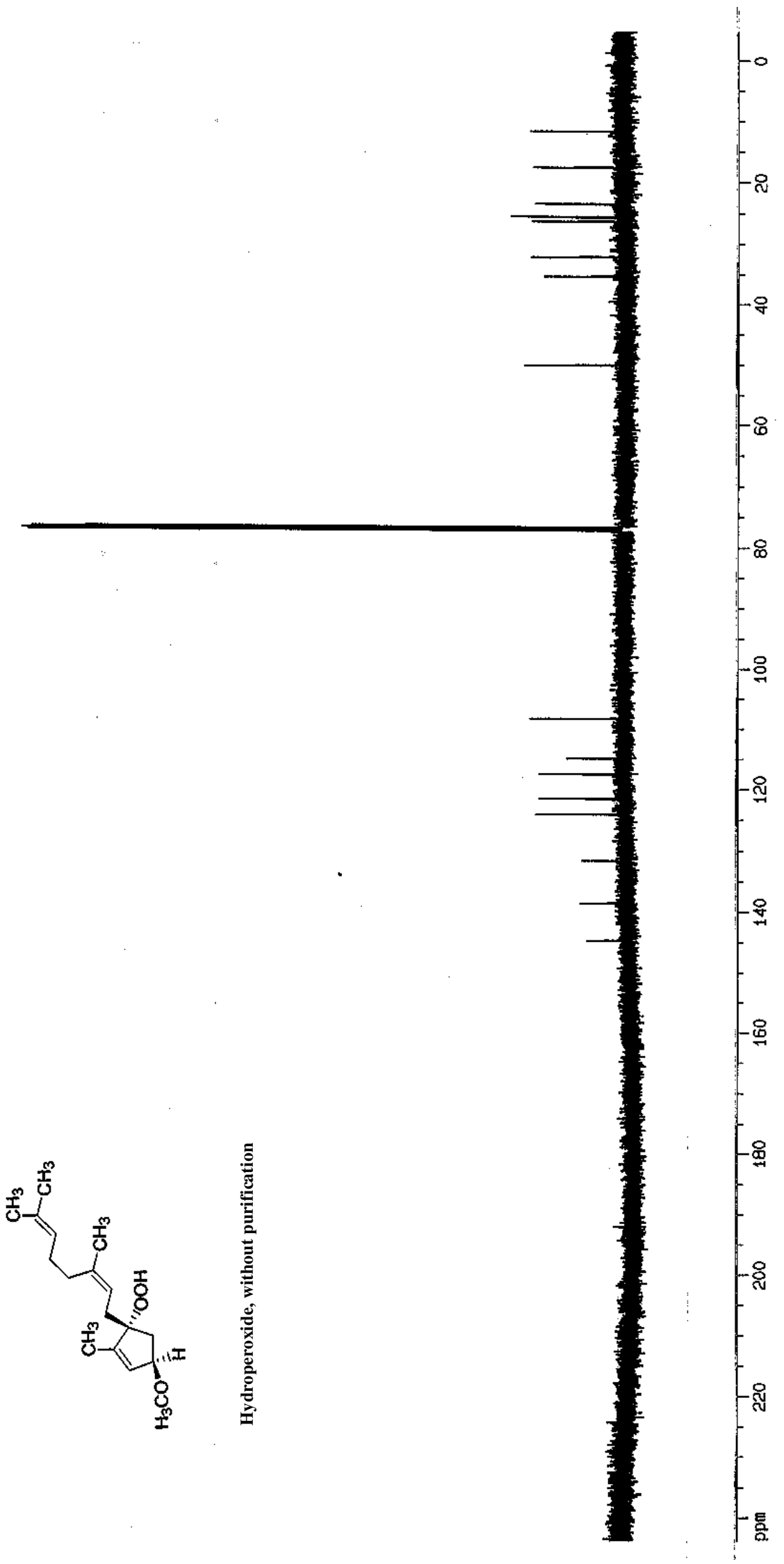

29E' $80 \mathrm{~T}$

898'b5 -

ET $\mathrm{G}^{\circ} \angle T$

009.52

$60 T^{\circ} \mathrm{bet}$

GL9' RET -...

$\angle 69^{\circ}$ BET-

It L'BOTL

undd 


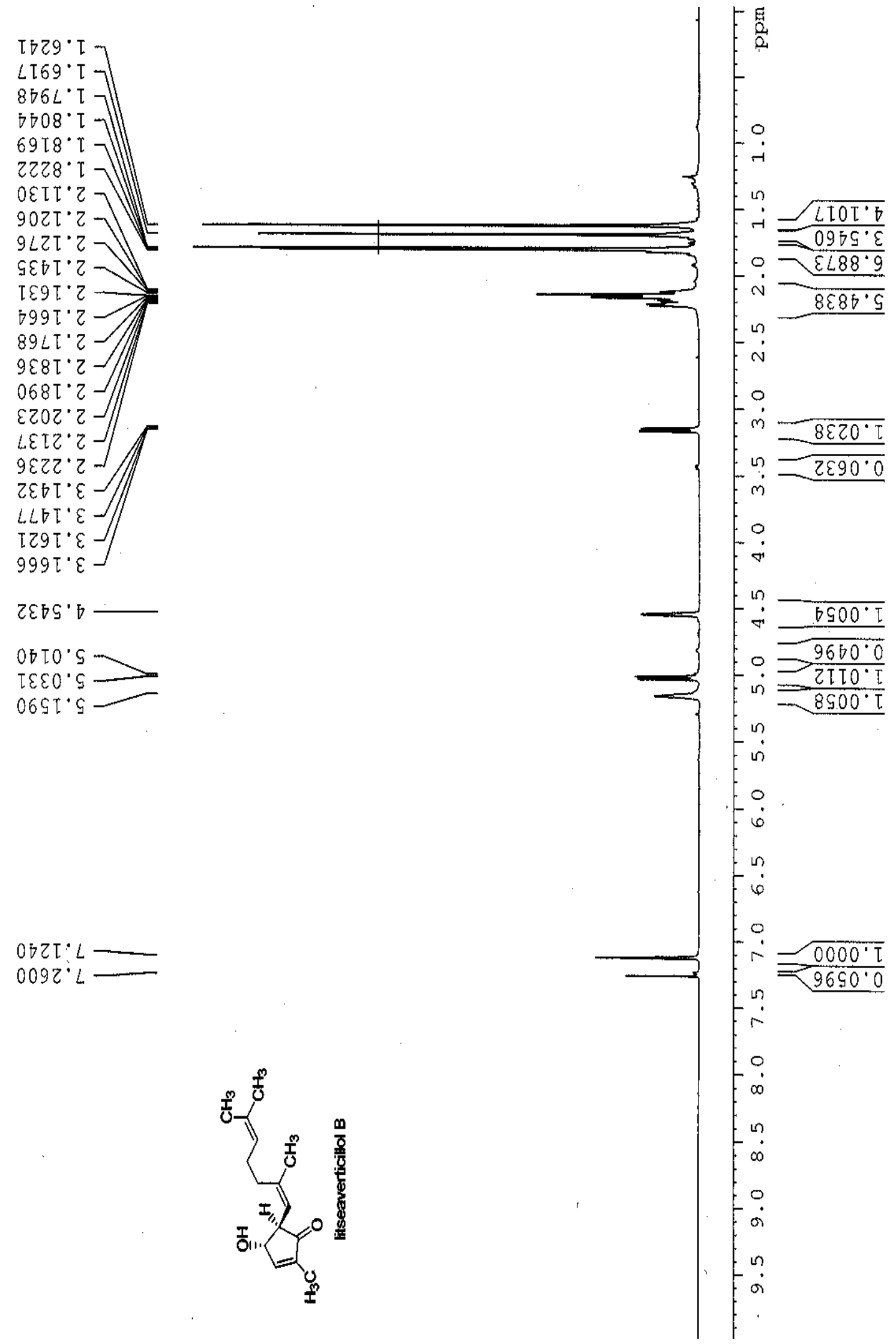


$\angle E C^{\circ}$ Or

$00 \angle \angle L=$

닽 $\varepsilon 2$

$\angle 89^{\circ} \mathrm{GC}$

E6G' 92

$\angle \forall G^{\prime} 己 \varepsilon$

سع

맏ㅇㄴ

$\angle b \angle \cdot 9 L$

$100^{\circ} \mathrm{LL}$

$\operatorname{sg} \cdot 4 L$

IDL' $6 I T-$

Ot6'EटT-

ZGE' ᄅEt-

$\nabla L L^{\prime} T V T=$

900. $201 \div$

$9 b \angle$ bGた
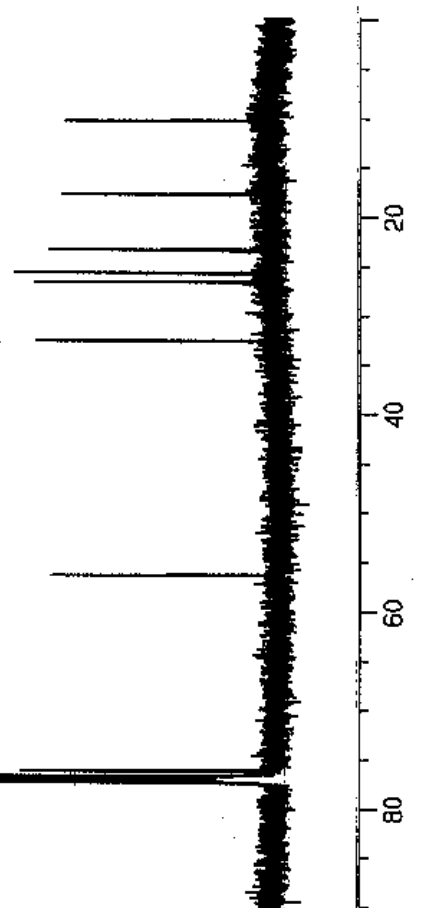

$6 E 2 \cdot 902-$

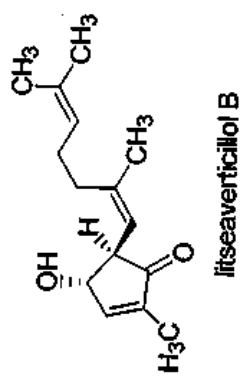

wdd 


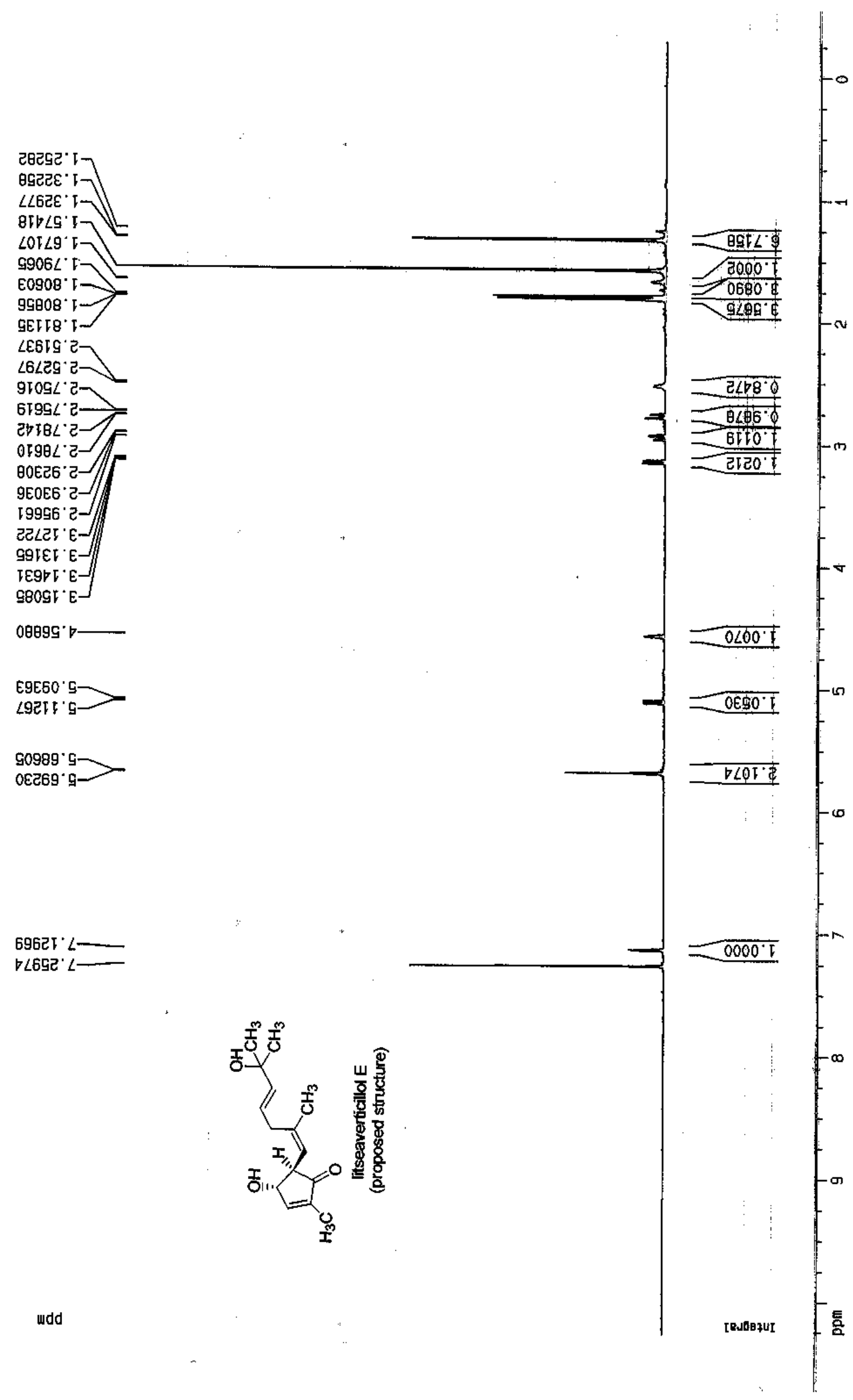



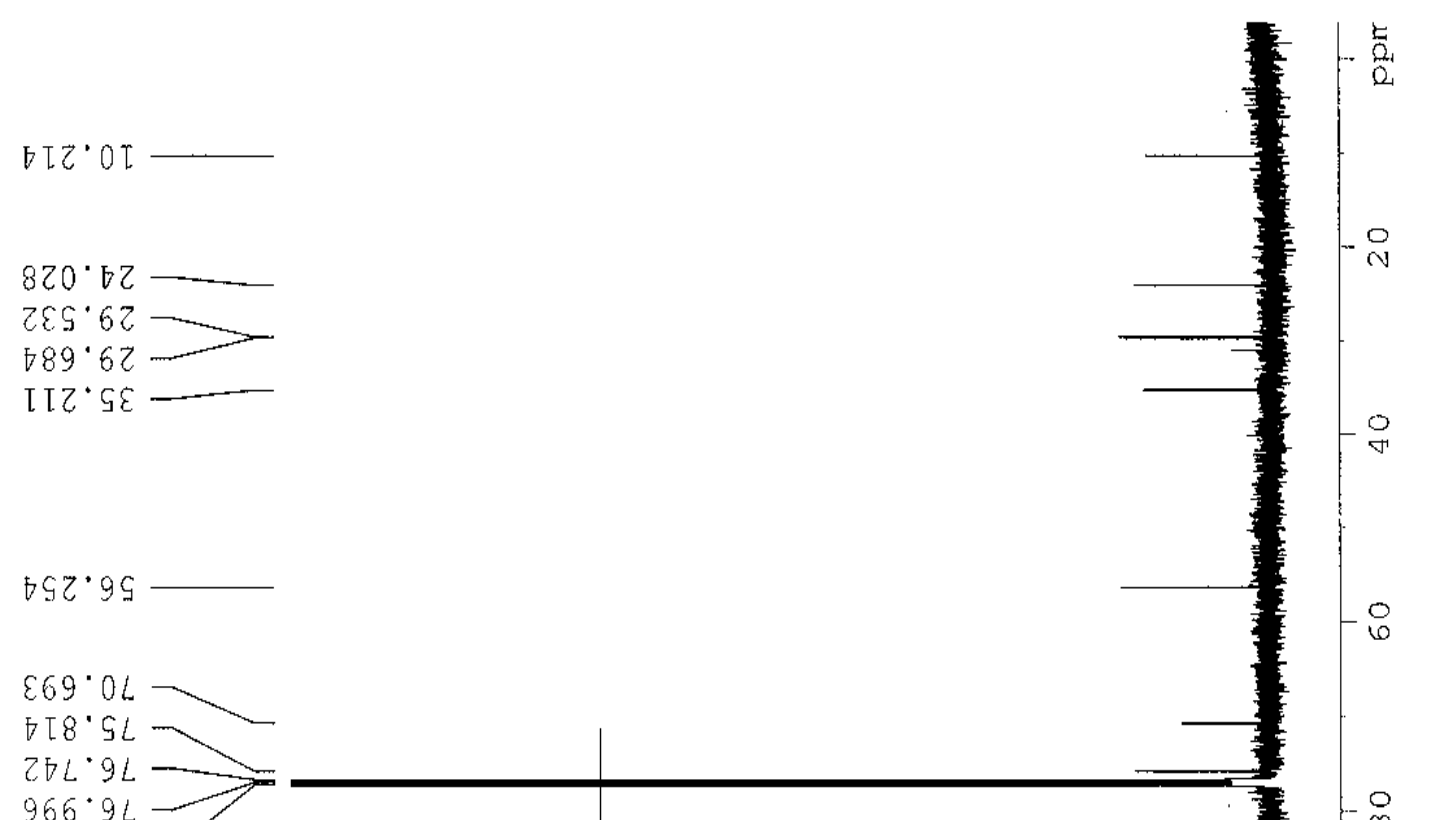

$296^{\circ} 02 \mathrm{I}-\ldots$

$626^{\circ} \mathrm{V2I}-$

$289 \cdot 8 \varepsilon \mathrm{I}-\mathrm{m}$

$099^{\circ} 68 \mathrm{~T} \longrightarrow$

$869^{\circ} \mathrm{ZtT} \longrightarrow$

$86 \mathrm{~T} \cdot \mathrm{SGT}$

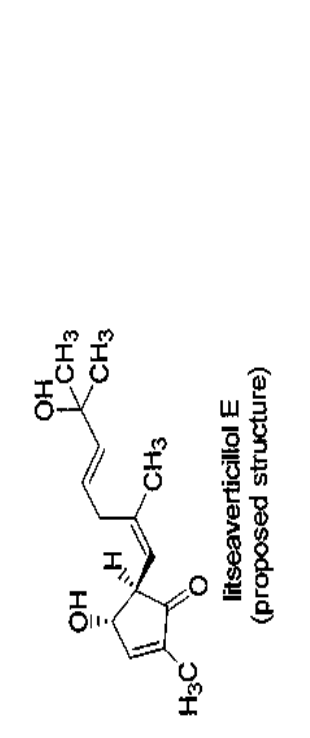

998.902 

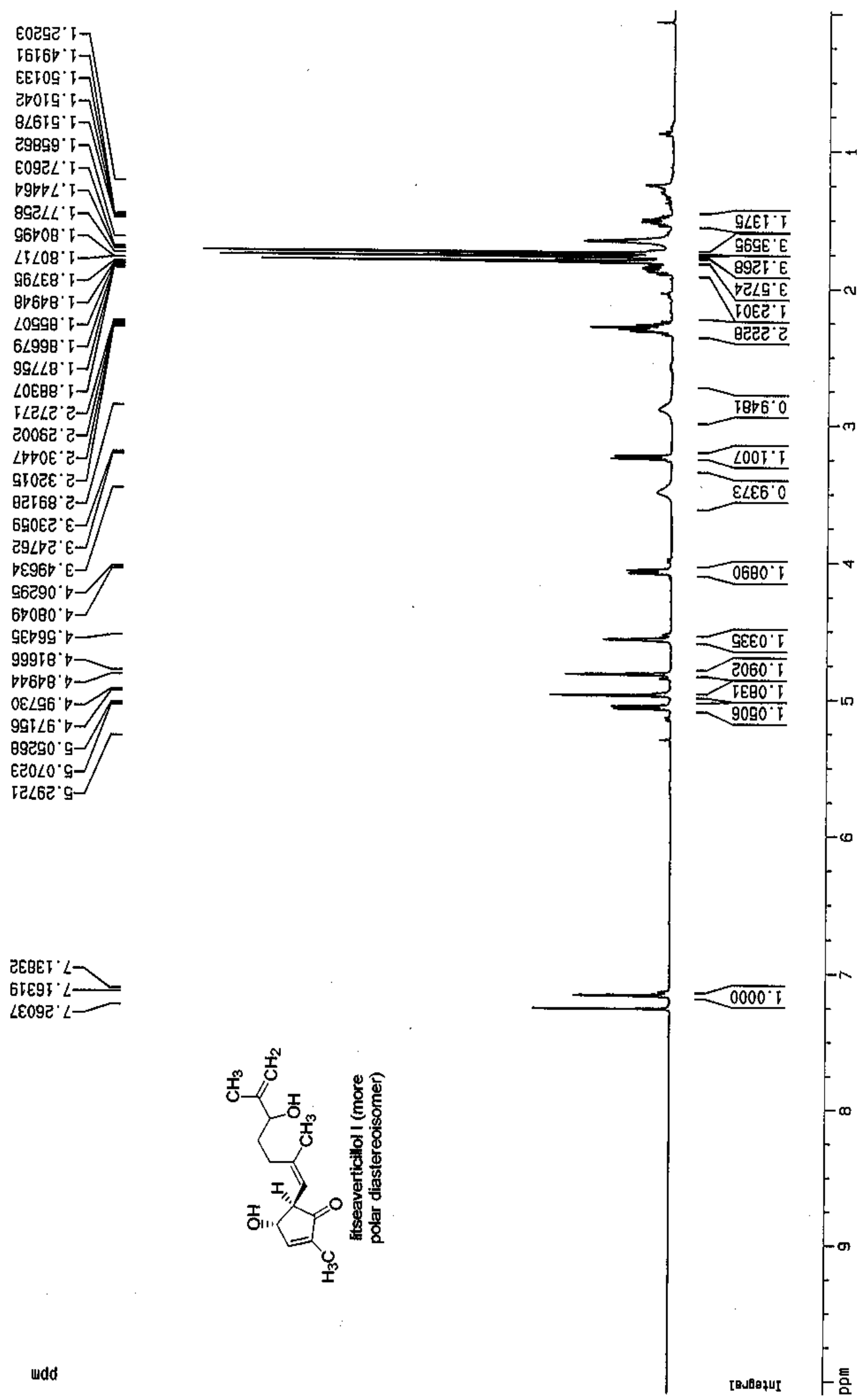


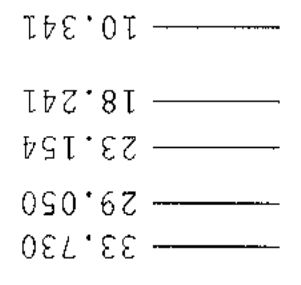

GT. 99

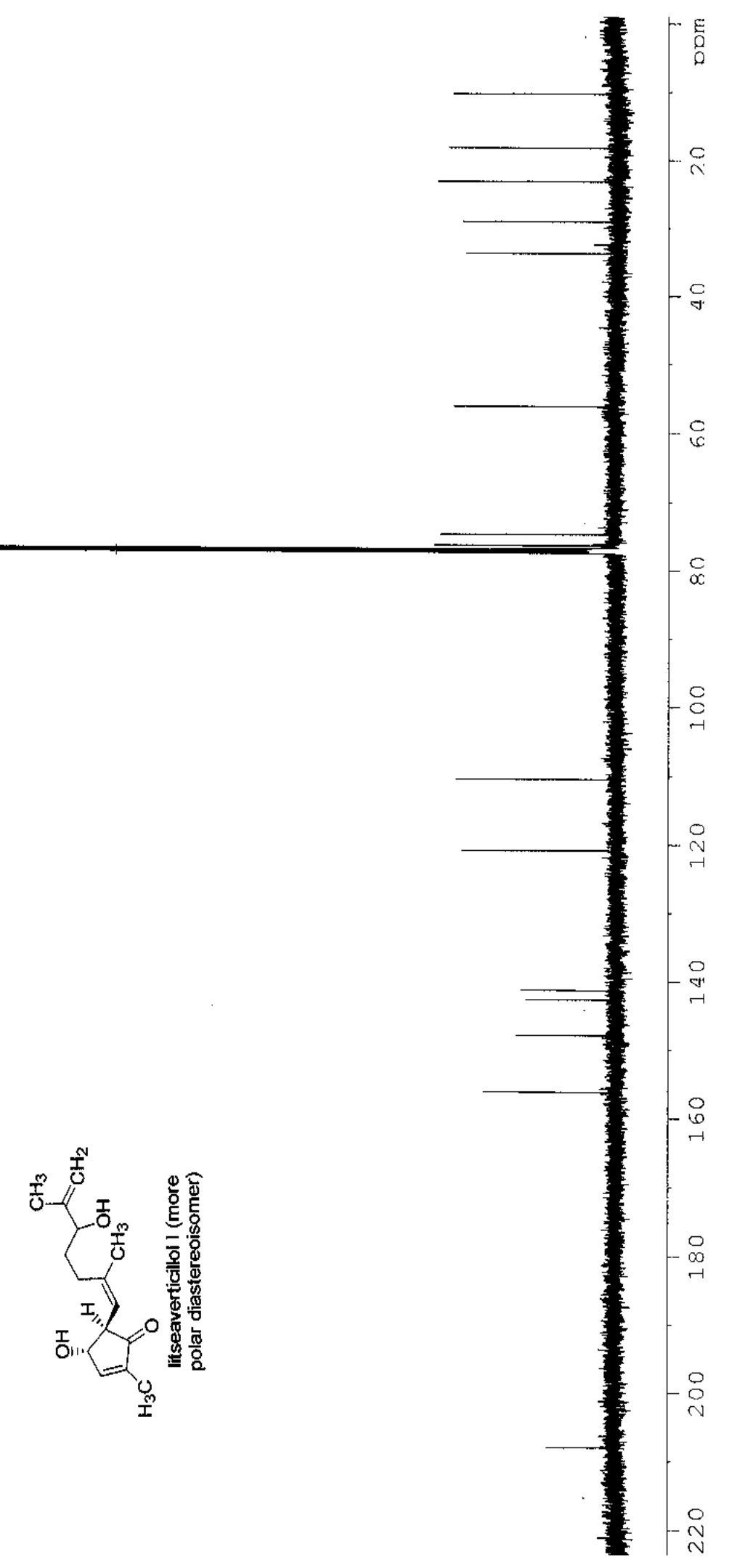

6TE't8T -

$869^{\circ} 20 \mathrm{~T} \ldots$

$8 \angle 6^{\circ} \angle F T$

$86 \tau \cdot 99 t$

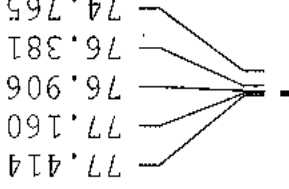

$\operatorname{LTO} \mathrm{S}^{\circ} \mathrm{L}$

$898^{\circ} 0 \mathrm{ZT}$

$900^{\prime} 80 z$ 
$\checkmark 229$

Gr9e9 $\mathrm{r}$

robs9' $\mathrm{F}-$

EBt99. 17

$\checkmark 206 \angle 9^{\circ} \mathrm{T}-$

6E2⿰日月 17

$\theta+9 E<1-1$

$0 \mathrm{OB} B \mathrm{C} \angle \mathrm{T}$

LES9L' ' -

62682

OBOta.

ธटеEв'



taibo'z-

$20890^{\circ} 2$

$99690^{\circ} \mathrm{z}-$

61995' $\mathrm{C}-$

$90+\angle 9$ ' 2

90189' $\mathrm{C}$

$89985^{\circ} \mathrm{c}-$

tr6te ${ }^{\circ} \mathrm{E}-$

ETEGE ' $E$ -

$66 \angle 9 C^{\prime} \mathrm{E}-$
$8 \angle \mathrm{L} \angle \mathrm{C} \cdot \mathrm{E}-$

TIBg6 $\mathrm{E}-$

$99700^{\circ} \mathrm{b}-$

GI629 ${ }^{\circ}$

6GZES '

gergo $\forall$

t5696.

Te6e T"

टtBg F $\mathrm{s}$

Gt $\angle 62 \cdot 5$

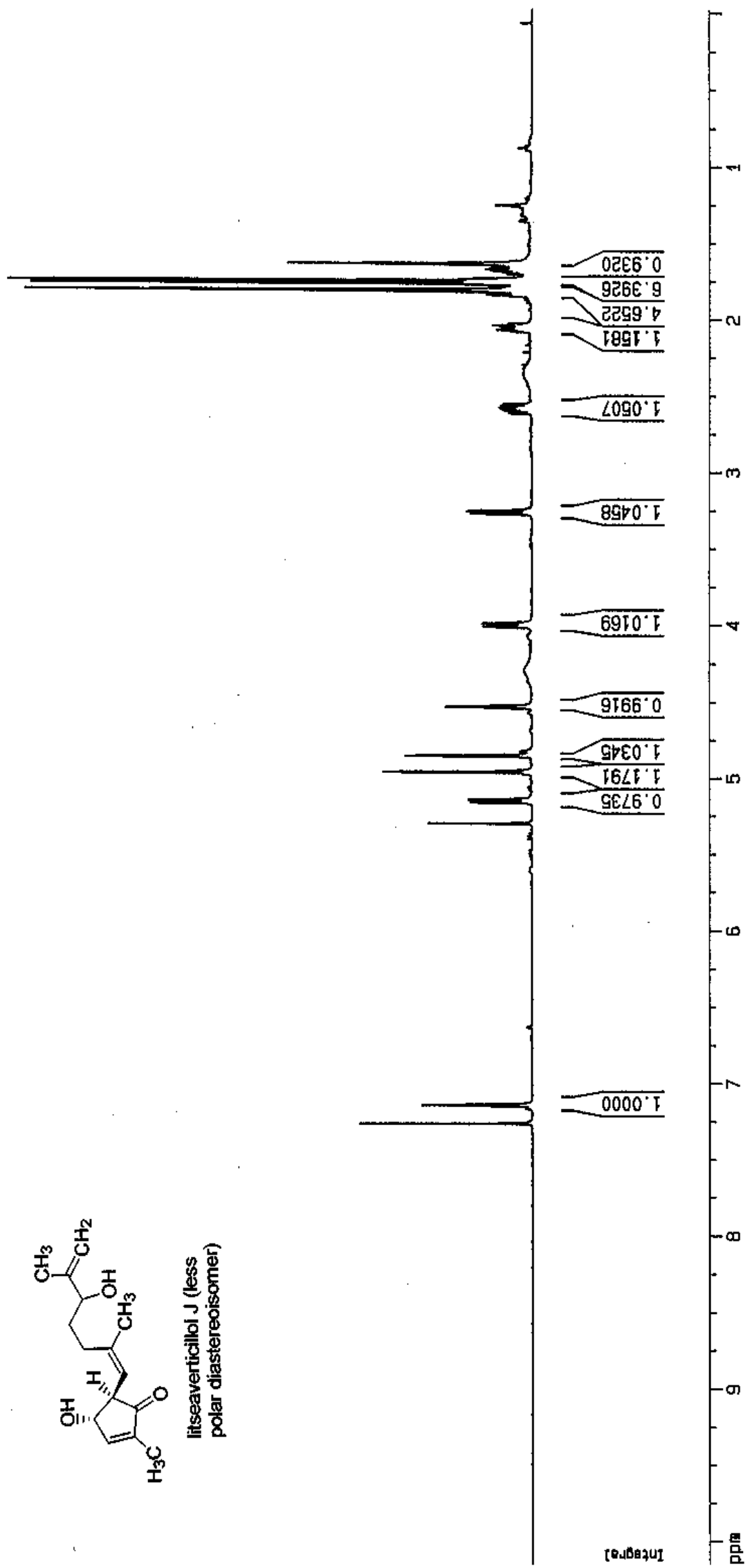




\section{$Z L E^{\circ} O T$}

$89 L^{\circ} 8 \mathrm{~T}$

$\varepsilon \varepsilon 8^{\circ} \mathrm{ZZ}$

$98 L^{\circ} L Z$

$\mathrm{S}^{\prime} \varepsilon \varepsilon^{\circ} \mathrm{Z \varepsilon}$

TZZ.99

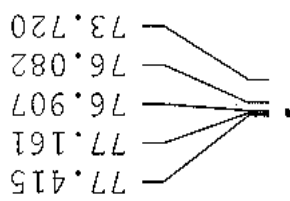

DOS OTt

$298^{\circ}$ LZL

$650.001-$
$89 L^{\circ} \mathrm{ZtL}-$
$089 \cdot \angle 6 T-$

$669^{\circ} \mathrm{g} \mathrm{I}$

I

$68 \varepsilon^{\circ} \mathrm{LOZ}$
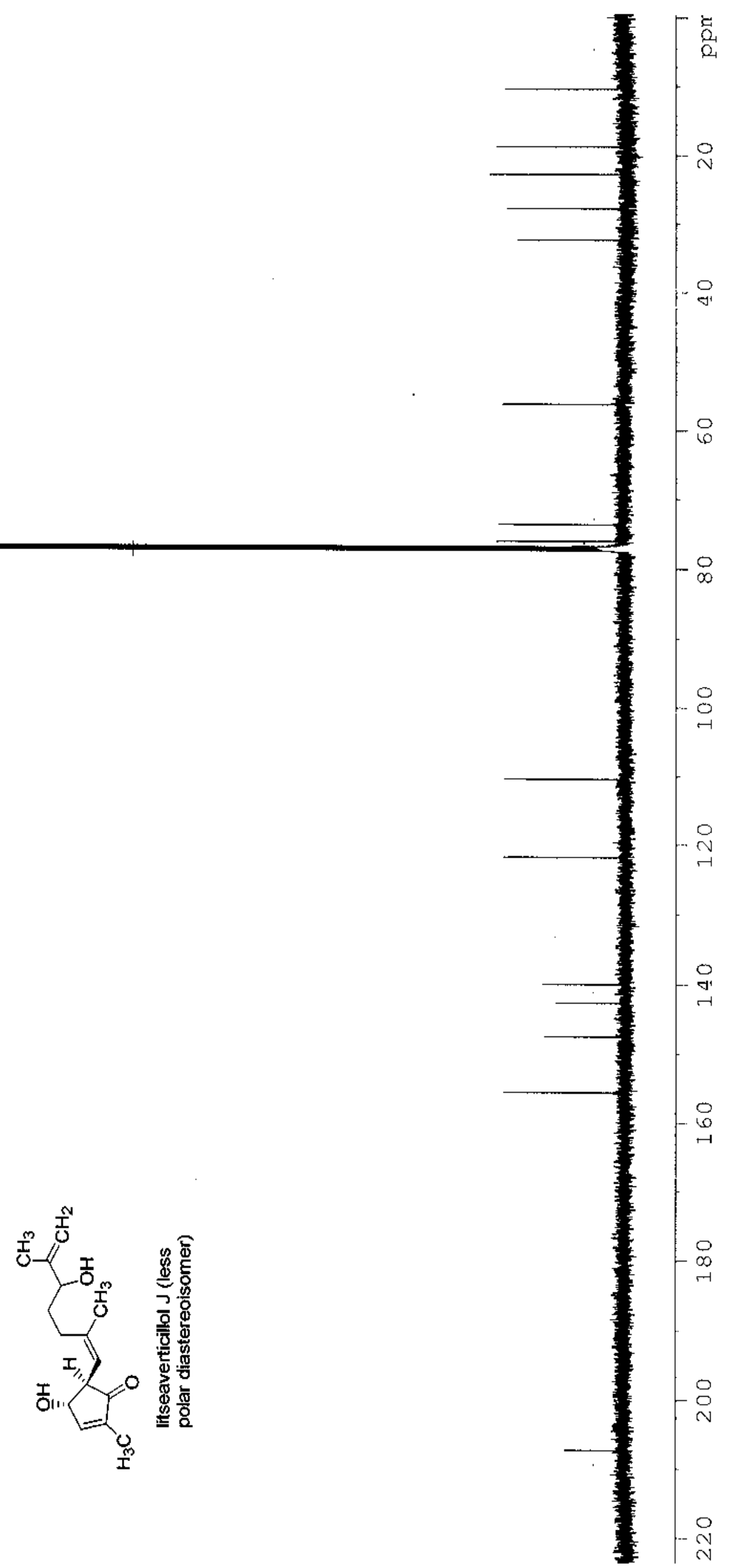


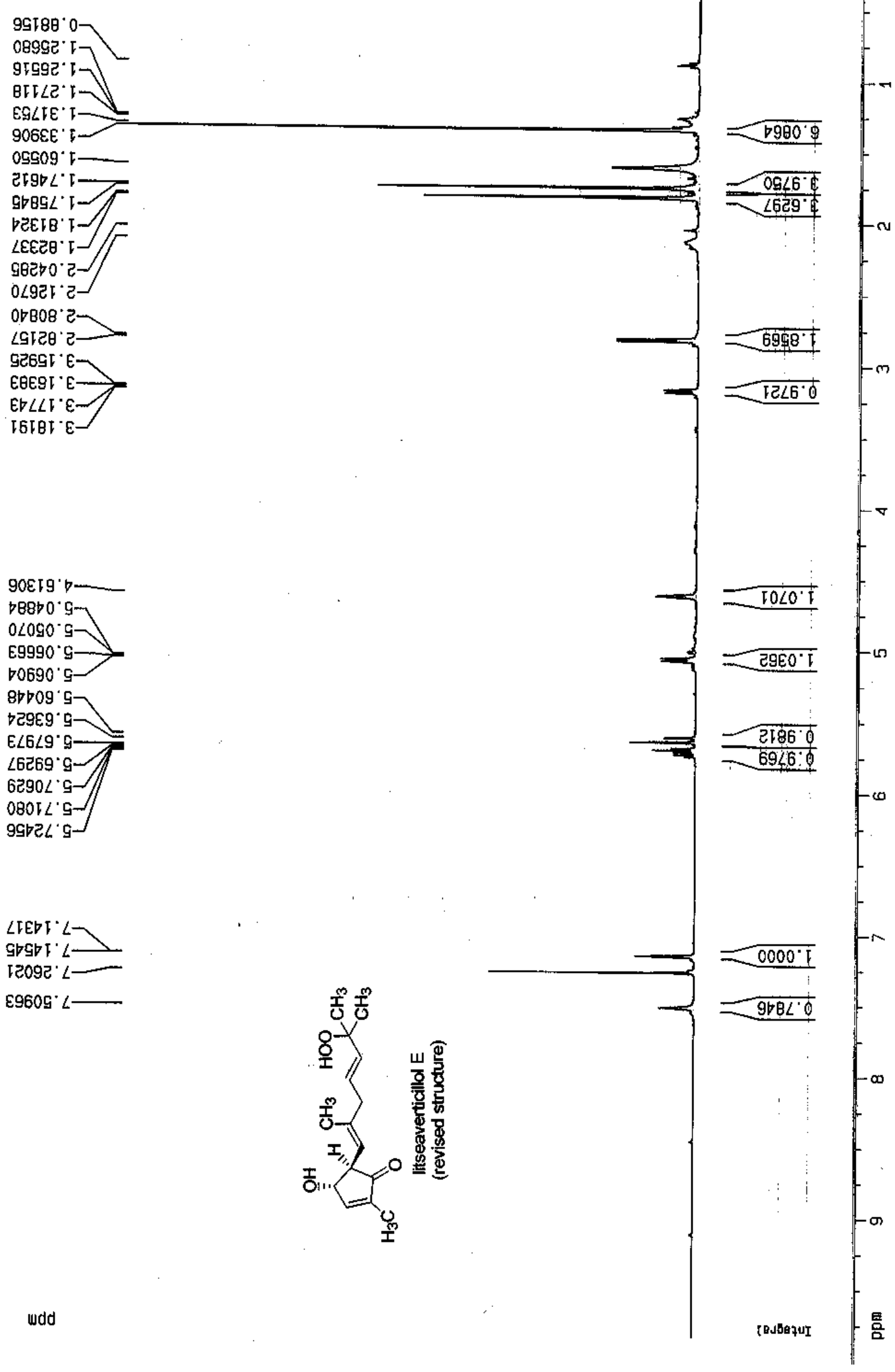



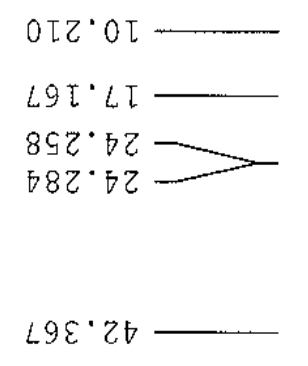

$462 \cdot 99-$

$882 \cdot 9 L$

$0 L \cdot 9 L \longrightarrow$
$\varepsilon 66 \cdot 9 L \longrightarrow=$

$8 \mp Z^{\circ} \angle L-$

$9 \mathrm{sT} 28$

$900^{\circ} 02 \mathrm{~T}$

$90 \mathrm{I} \cdot 62 \mathrm{~T}$

$\varepsilon \varepsilon \nabla^{\circ} \mathrm{GL \textrm {T }}$

$\mathrm{T} \nabla \nabla^{\circ} \circ \forall \mathrm{T}$

$208^{\circ} \mathrm{ZVT}-$

$686^{\circ} \mathrm{DSI}$

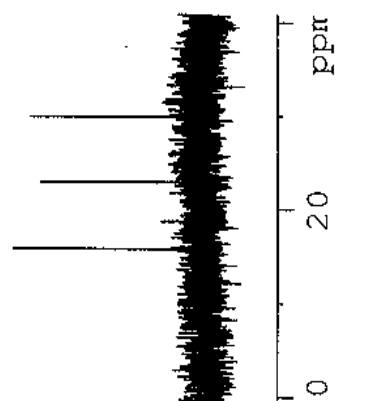

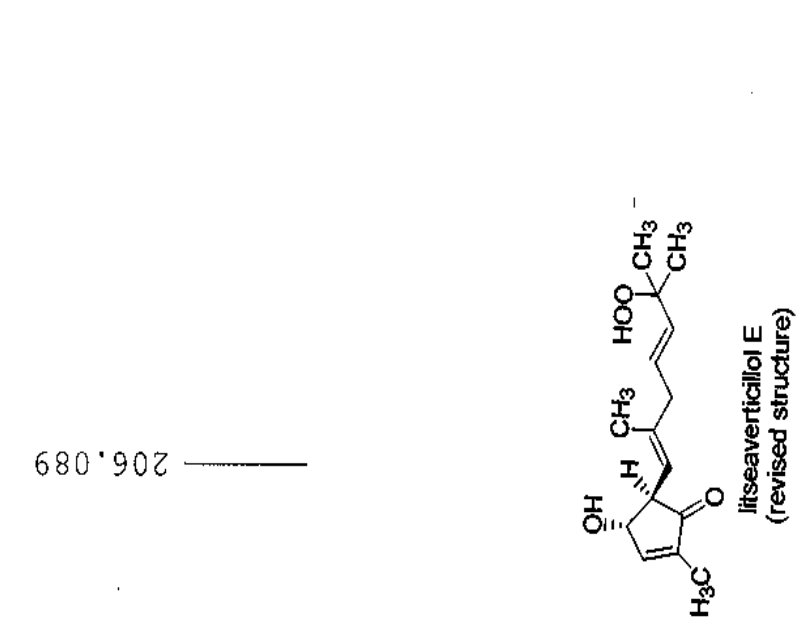

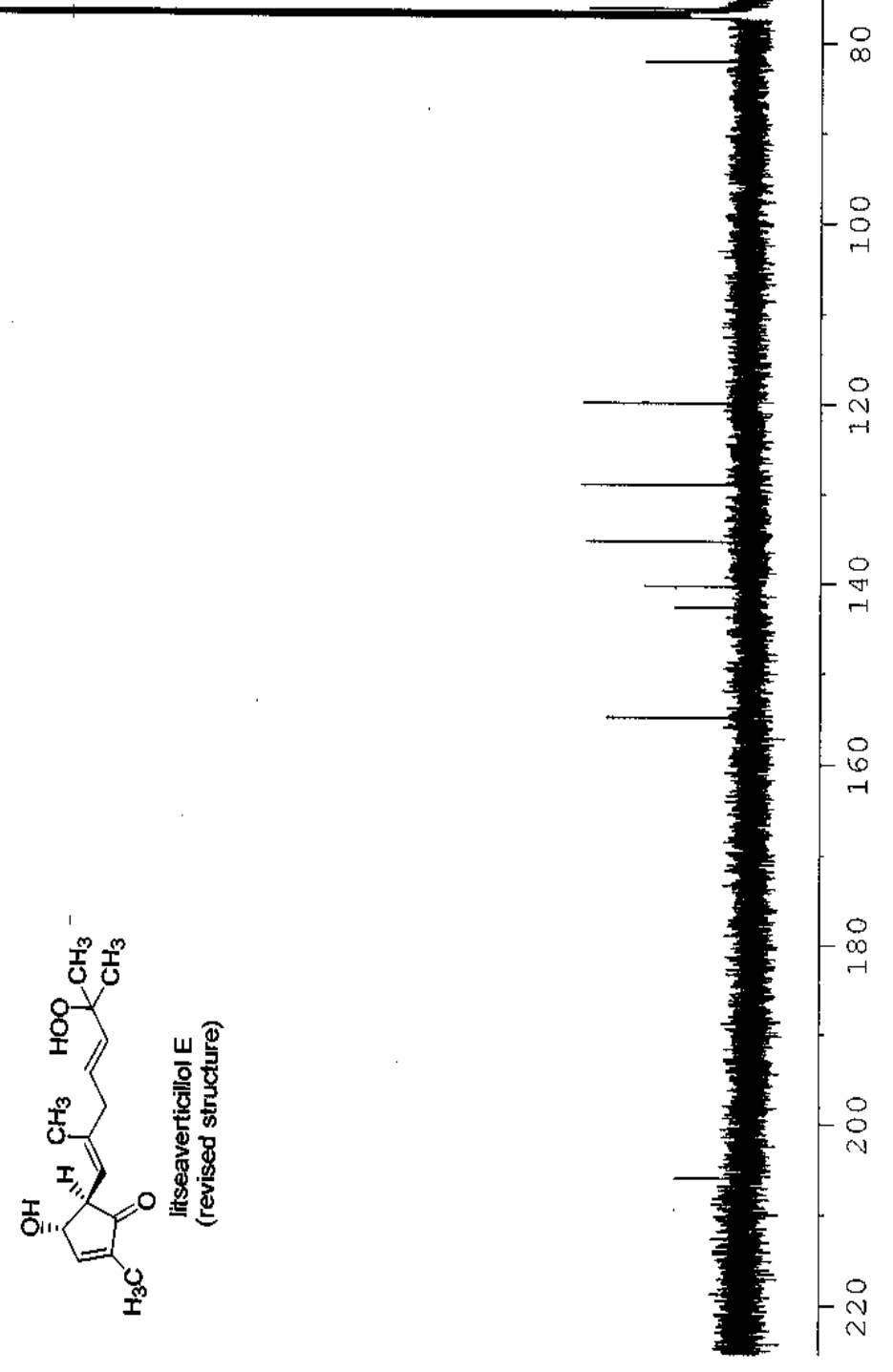



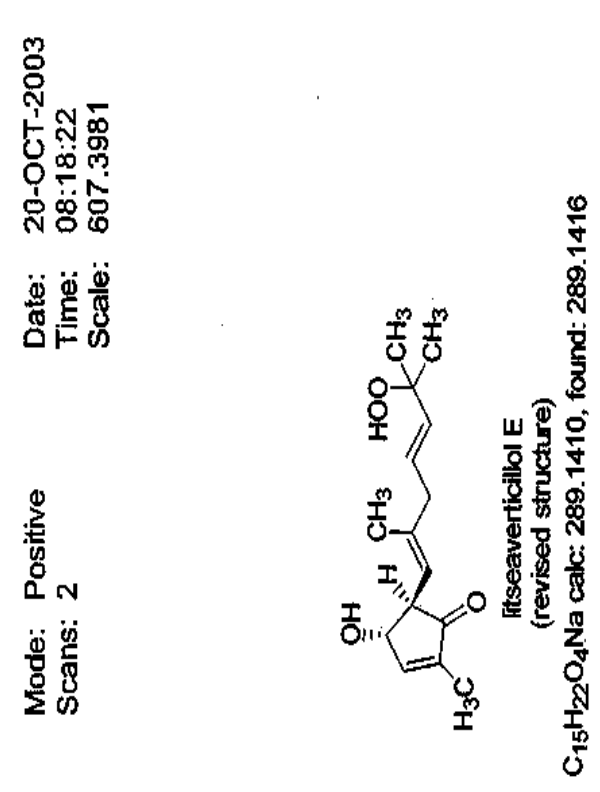

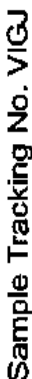

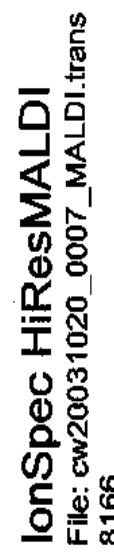

范
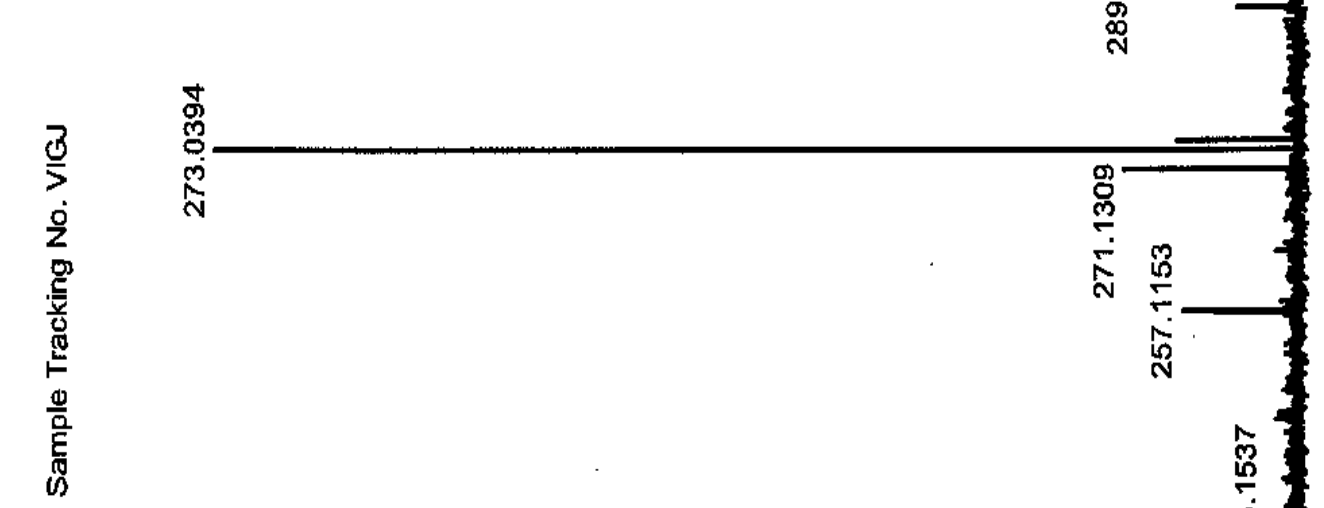